\title{
Xeno-free and feeder-free culture and differentiation of human embryonic stem cells on recombinant vitronectin-grafted hydrogels
}

\begin{abstract}
Recombinant vitronectin-grafted hydrogels were developed by adjusting surface charge of the hydrogels with grafting of poly-l-lysine for optimal culture of human embryonic stem cells (hESCs) under xeno- and feeder-free culture conditions, with elasticity regulated by crosslinking time $(10-30 \mathrm{kPa})$, in contrast to conventional recombinant vitronectin coating dishes, which have a fixed stiff surface $(3 \mathrm{GPa})$. hESCs proliferated on the hydrogels for over 10 passages and differentiated into the cells derived from three germ layers indicating the maintenance of pluripotency. hESCs on the hydrogels differentiated into cardiomyocytes under xeno-free culture conditions with much higher efficiency $\left(80 \%\right.$ of $\mathrm{cTnT}^{+}$cells) than those on conventional recombinant vitronectin or Matrigel-coating dishes just only after 12 days of induction. It is important to have an optimal design of cell culture biomaterials where biological cues (recombinant vitronectin) and physical cues (optimal elasticity) are combined for high differentiation of hESCs into specific cell lineages, such as cardiomyocytes, under xeno-free and feeder-free culture conditions.
\end{abstract}

